\title{
II. BASIC DATA AND CALIBRATION PROBLEMS
}

\section{A. Basic data}

An important development during the triennium under review has been the creation of a center at Strasbourg for the collection and organization of astronomical data. This new Centre de Données Stellaires publishes information of value for many problems of stellar statistics and galactic research. Reports in the form of Information Bulletins have already appeared (Jung 05.002.043; 06.002.026; 07.002.020).

Woolley et al. (04.041.011) have presented a wealth of information concerning the stars within $25 \mathrm{pc}$ of the Sun. Gliese (02.041.018) also has published a catalogue of stars nearer than $22 \mathrm{pc}$. A catalogue containing data for low luminosity stars summarizes the work of many years by Luyten (03.126.008) in which through proper motion surveys he has detected stars of luminosity less than 0.001 that of the Sun.

Among the catalogues of stellar data we also mention those by Stephenson and Sanduleak (06.041.023), Wackerling (04.114.004), Bertiau and McCarthy (02.114.046), and Westerlund (06.114.013). Stephenson now has underway a general catalogue of carbon stars. It will contain a listing of all carbon stars having published equatorial coordinates together with data for some new carbon stars detected in the surveys at the Warner and Swasey Observatory.

A useful catalogue and bibliography of B type emission stars has been published by Jaschek $e t$ al. (06.114.129). As a continuation of the listing in Mt. Stromlo Mimeograms No. 7 of O-B5 stars Buscombe (1972) has provided in Mimeograms No. 8 a summary of photometric and spectroscopic data for supergiants of later spectral classes.

A list of MK spectral classifications for 600 southern hemisphere stars of types K2V and later and brighter than $m_{v} \sim 10$ has been published by Upgren et al. (1972). These supplement the list of $\mathrm{dM}$ and dK stars (some $895 \mathrm{stars}$ ) observed years ago at the McCormick Observatory for $\delta>-30^{\circ}$.

The Henry Draper Catalogue and Extension has long been basic for data on spectral types, positions and approximate photometry. It contains, however, no systematic luminosity classifications for the stars. To remedy this, to modernize the vast store of useful data for stars principally brighter than $9.5 \mathrm{mag}$, a program of two-dimensional spectral classification of all HD stars south of $\delta=+30^{\circ}$ is underway at the University of Michigan by Houk and Cowley. In order to assure as uniform a system as possible all classifications are being made by Houk (06.114.055), with frequent crosschecking by other experts. It is estimated that all stars south of $\delta=-55^{\circ}$ will be classified by late 1972.

Infrared observational data for approximately 5600 objects have been provided by Neugebauer and Leighton (1969). A catalogue of polarization measurements for 1660 southern OB stars has been published by Klare et al. (07.131.011). Becker and Fenkart (06.153.015) have provided a useful list of 216 open clusters giving position, interstellar absorption, distance, diameter and references for each.

Kukarkin reports that the preparation of a General Catalogue of Globular Clusters is progressing but that some delay has been caused by the problem of diameters. Revisions of apparent distance moduli are based on RR Lyr stars and their luminosities according to calculations by Christy. The distance scale obtained is slightly smaller than that now used.

Lynga and Hansson (1972) have recently published an atlas of nebulae for the southern Milky Way. Another useful atlas has been provided by Haffner and Nowak (01.041.020).

One most interesting report is that entitled The Scientific Results from the Orbiting Astronomical Observatory (OAO-2) (Code 1972). This contains many papers of interest to students of galactic structure.

A Catalogue of Stars Observed Photoelectrically by C. Jaschek, E. Hernandez, A. Sierra and A. Gerhardt has now been completed at La Plata and is in press.

We review now, briefly, the main radio surveys which have covered a major part of the sky or of the Milky Way region. Many details concerning these surveys will be found in the report of Commission 40. 
In the 21-cm hydrogen line, there have been three large surveys of the Milky Way strip. In the north, Westerhout $(03.157 .028 ; 1973)$ has obtained a very detailed coverage in a narrow range of latitude (from $b= \pm 1^{\circ}$ to $b= \pm 3^{\circ}$ ) with the $10^{\prime}$ beam of the Green Bank 91-m telescope in the longitude range $11^{\circ}-235^{\circ}$, and Weaver $(03.155 .028 ; 1972)$ has covered a broader strip $\left(b= \pm 10^{\circ}\right)$ with the Hat Creek $26-\mathrm{m}$ telescope in the range $l=10^{\circ}-250^{\circ}$. In the south, the range $l=185^{\circ}$ through $360^{\circ}$ to $63^{\circ}$ has been surveyed in a less complete manner with the Parkes 64-m telescope [Kerr (02.157.002); Hindman and Kerr (04.155.036); Kerr and Hindman (04.155.034)].

Three groups have obtained $21-\mathrm{cm}$ profiles for a large number of points spread over the whole sky visible from a northern observatory. Tuve and Lundsager $(1972 \mathrm{a}, \mathrm{b})$ have concentrated on low latitudes from $l=336^{\circ}$ to $270^{\circ}$, Venugopal and Shuter (04.157.001) have published 1047 profiles for a $5^{\circ}$ grid, and Habing and Heiles (1971) have recorded profiles over the whole sky observable with the Hat Creek 26-m telescope. Measurements of 21-cm absorption have been made by Radhakrishnan et al. (07.131.002; and .003), Goss et al. (07.131.004), Hughes et al. (06.141.192), and Kerr and Knapp (04.155.035).

In the radio continuum, general surveys of the galactic radiation have been carried out by Berkhuijsen (07.157.004), Hamilton and Haynes (02.157.019), Landecker and Wielebinski (04.157.012), and Rohan and Soden (03.055.025). The most extensive survey of linear polarization is that by Spoelstra (07.157.003) at $1415 \mathrm{MHz}$. Two very detailed surveys of the continuum emission at low latitudes have been presented in papers listed by Day et al. (03.157.014), and by Altenhoff et al. (03.157.012). Several other studies have been concerned with more limited regions of the sky.

Several hundred planetary nebulae have been observed at radio wavelengths; however, only a small fraction, perhaps 100 , have reliably determined fluxes at one or more wavelengths. The subject has been extensively reviewed by Higgs (06.133.032) and Heckathorn (05.133.027).

Surveys concerned with the over-all galactic distribution of $\mathrm{OH}$ sources have been described by Robinson et al. (03.131.123), Goss et al. (04.141.102), Manchester et al. (04.131.116), Robinson et al. (06.131.069), and Turner (04.131.007; 07.131.023).

Two extensive galactic surveys of $\mathrm{H} 109 \alpha$ recombination-line emission from $\mathrm{H}$ II regions have been reported, by Reifenstein et al. (03.157.011) for the northern sky and Wilson et al. (03.131.095) for the southern sky. The subject has been reviewed by Dupree and Goldberg (04.131.058).

\section{B. Intrinsic colors and interstellar reddening}

The intrinsic colors of highly luminous stars and the ratio of interstellar absorption to reddening, two important desiderata for determining stellar distances photomertically, are considered in this section.

Mean intrinsic colors on the $U B V$ and Cape $U_{\mathrm{c}} B V$ systems have been published by FitzGerald (03.113.018). Morgan et al. (05.153.006) have given revised spectral types (MK70), and UBV pe data for some 112 stars of the $\alpha$ Per cluster which have yielded intrinsic colors for the main sequence (classes IV and V). On the average these differ by only $0.01 \mathrm{mag}$ from the values of $(B-V)_{0}$ given by Johnson (1966). Häggkvist (05.113.014) has derived intrinsic colors from a calibration of an $\mathrm{H} \gamma$ absorption index by narrow-band photometry for bright stars earlier than A2 in the northern hemisphere. Häggkvist and Oja (03.113.025) obtained relations yielding $(B-V)_{0}$ as a function of their measured local color indices for $\mathrm{G}$ and $\mathrm{K}$ stars.

An extensive study by Lee (04.113.038) of high luminosity M-type stars by multi-color photometry has yielded intrinsic color indices, bolometric corrections and effective temperatures. Wawrukiewicz (1970) has devised a narrow-band photometric index to obtain the spectral type of $M$ giant and supergiant stars. This together with $V, R$ photometry has been applied to the determination of intrinsic colors for $M$ giants and supergiants. He finds intrinsic colors in good agreement with those found by Lee.

Stromgren (02.114.112) has summarized efforts at calibration of the uvby $\beta$ photometric system and its applications. Crawford $(03.113 .040 ; 06.113 .039)$ has given a brief summary of his extensive work on intrinsic colors and the evaluation of interstellar extinction in this photometric system.

An article by Johnson (1968) reviews the status of interstellar extinction and its variation with 
position in the Galaxy. Rossiger (07.131.020) has applied a two-color star count method for deducing the values of $R$ for several interstellar clouds and finds values ranging from 3.1 to 4.4 with mean errors of \pm 0.3 to \pm 0.6 . New and highly accurate spectral types for 84 stars in the association III Cep at $l=110^{\circ}, b=+2^{\circ}$ together with $U B V$ photometry have led Garrison (04.152.006) to conclude that $R$ in this region has a most probable value 3. In a study of evaporation processes surrounding early type stars Isobe (06.152.002) has evaluated in detail the ratio $R$ for several subregions of the Orion complex.

Bell and FitzGerald (1971) and Crézé (1972) have used kinematic methods based upon radial velocity data to determine $R=A_{\mathrm{v}} / E_{B-V}$. In a similar analysis Martin (06.155.001) has assumed a galactic rotation model of the Galaxy together with observed radial velocity data to derive $A_{V} / E_{B-V}=3.3 \pm 0.6$ and a distance to the galactic center $R_{0} \sim 9 \mathrm{kpc}$.

Seggewiss reports that Wiemer has started a program to redetermine the mean law of interstellar extinction for $O$ type stars and B type supergiants. Broadband photometry from the ultraviolet to the infrared $(0.35 \mu \leqslant \lambda \leqslant 3.5 \mu)$ is used.

It would appear from present knowledge of intrinsic $U B V$ colors corresponding to MK spectralluminosity-classes, that the usual ratio $R=3$ to 3.2 can be safely adopted in most applications.

\section{Absolute magnitudes}

The calibration of the HR diagram and the determination of absolute magnitudes, together with estimates of their accuracy and cosmic dispersion, provide much necessary data for investigations of galactic structure. Stars within $25 \mathrm{pc}$ of the Sun have been studied extensively by Woolley et al. (06.155.007). In addition to the kinematic data (summarized in Section V of this Report) the authors have provided the values of $M_{\mathrm{v}}$ for the main sequence stars A0V to M5V. Comparison with the Hyades indicates only minor differences. The mean $M_{v}$ for a given spectral type is estimated to be accurate to about $0.1 \mathrm{mag}$.

Gliese (05.115.015) discusses the relationships between $U B V$ colors and spectral types, and gives a calibration of $M_{\mathrm{v}}$ versus MK spectral type for classes F5V to M2V for stars nearer than $22 \mathrm{pc}$. The average absolute difference in $M_{\mathrm{v}}$ between the main sequence calibrations by Gliese and by Woolley et al. is only 0.04 mag over the spectral class range F0 to M2.

Blaauw (07.111.006) has discussed the application of statistical parallaxes to the calibration problem and concludes that probable errors of 0.2 to $0.3 \mathrm{mag}$ for $A$ and $F$ main sequence and giant sequence stars are attainable at present.

A new method of treating stellar motions was introduced by Upton (04.153.038), who used it to determine the distances of the Hyades and Praesepe clusters. Agreement with the distance modulus of the Hyades obtained by the convergent point method is good. Mannery and Wallerstein (06.153.017) have compared photometric data for 88 field stars with $\pi>0$ " 08 and 58 Hyades stars to obtain a modulus of $3.24 \mathrm{mag} \pm 0.1$ (m.e.) for the cluster which compares favorably with $3.23 \pm 0.05$ mag obtained by Van Altena (01.153.020). Eggen (02.153.029) has compared by $(R, I)$ photometry 24 red dwarf stars in the Hyades cluster with 25 field stars of the young disk population which have large trigonometric parallaxes and finds that the Hyades main sequence defined by the convergent point method, and the main sequence defined by the stars of known parallax differ by less than $0 \cdot 1$ mag.

Several calibrations of $M_{\mathrm{v}}$ versus the MK spectral-luminosity class for supergiant stars have been made during the triennium under review. Walborn (07.114.071) has deduced from new data for stars of classes $\mathrm{O} 3$ to $\mathrm{B} 2.5$ in 14 clusters absolute magnitudes $M_{\mathrm{v}}$ which are in agreement with the calibration by Weaver and Ebert (1964). In a similar analysis Stothers (1972) has calibrated $M_{\mathrm{v}}$ for massive supergiants (classes Ia, Iab, Ib) from cluster and association membership.

Crampton (07.115.003) has compared the luminosities of 49 stars in the Perseus spiral feature derived on the basis of MK spectral types with those derived on the basis of an $\mathrm{H} y$ equivalent width calibration. The two calibration systems appear to be consistent. Crampton finds the random errors reduced to about $0.3 \mathrm{mag}$ by taking means of the $\mathrm{MK}$ and $\mathrm{H} \gamma$ absolute magnitudes. 
Calibrations of $M_{\mathrm{v}}$ for supergiants of later spectral types have been made by Bouw and Parsons (1971) for stars of spectral-luminosity classes A0 through G0, Iab and Ib, with a few Ia; by Keenan (05.114.081) for $G, K$ and $M$ supergiants and for high luminosity $M$ stars by Lee (04.113.038).

The absolute magnitudes of Wolf-Rayet stars have been evaluated by Rublev (04.115.007) with results which are in good agreement with the calibration by Smith (1968). In two papers dealing with WR stars and OB stars in association with $\mathrm{H}$ II regions Crampton $(05.114 .048 ; 06.114 .015)$ has concluded that the $M_{v}$ calibration for WR stars by Smith should be corrected by +0.8 mag.

Statistical methods for calibrating the main and the giant sequences of the HR diagram have been discussed extensively by Jung $(03.115 .002 ; 05.115 .003)$. Tables give mean $M_{\mathrm{v}}$ and $\sigma_{\mathrm{M}}$ as functions of MK spectral class for main-sequence and giant branches of the HR diagram.

Morgan et al. (05.153.006) have used newly revised spectral-luminosity types and UBV photometry to establish a well defined main-sequence from B3 to G0 for the $\alpha$ Per cluster. The main sequence between F2 and G0 is extremely sharply defined. For the main sequence from B0.5 to B9 Murphy (02.118.028) has derived an absolute magnitude calibration from 81 binary systems in which one component is of class $B$.

While calibrations of luminosity by narrow-band or intermediate band photometry are more appropriately the province of Commission 25, the usefulness of these techniques for studies of galactic structure cannot be overemphasized. These have been reviewed by Strömgren (02.114.112) and by Crawford (1972). Haug (04.115.012) has derived by $\mathbf{H} \beta$ photometry absolute magnitudes for OB stars.

A calibration of the photometric parameters in the uvby $\beta$ and the $U B V$ systems from data for about 1000 bright $F$ stars by Eggen (06.115.017) yields a ZAMS for $+2.55<M_{\mathrm{v}}<+5.1$ with an accuracy of about $0.3 \mathrm{mag}$ in $M_{\mathrm{v}}$. The errors are estimated to be of the order of 0.4 to $0.5 \mathrm{mag}$. Two investigations, by Häggkvist (05.113.014) and by Häggkvist and Oja (03.113.025), provide narrow-band photometric calibrations of $M_{\mathrm{v}}$. A calibration of the multicolor photometric system employed at Vilnius has been given by Sviderskiené and Straizys $(05.115 .018 ; 07.115 .007)$.

\section{The stellar luminosity function}

Two recent investigations concerning the general stellar luminosity function have raised questions about the value of $M_{\mathrm{v}}$ or $M_{\mathrm{b}}$ at which the maximum frequency $\phi(M)$ occurs, and about the function $\phi(M)$ itself for $+10<M<+15$. A preliminary analysis by Luyten (1968) of his extensive proper motion data indicated that $\phi\left(M_{\mathrm{b}}\right)$ had a maximum at $M_{\mathrm{b}}=+15 \cdot 7$. Recently, however, Wanner (07.115.001) has questioned the statistical methodology used by Luyten and has concluded that the absolute magnitudes assigned are too low (stars too bright) by at least a full magnitude, especially near the frequency maximum. Wanner finds a maximum at $M_{\mathrm{b}}=+12$, about 3 mag brighter than Luyten's value. The conflict is self-evident.

The other investigation, by Murray and Sanduleak (07.112.013), leads to a preliminary conclusion that the population of faint dwarf $M$ stars near the galactic plane is much larger than thought heretofore. If so, the values of $\phi(M)$ for $+10<M<+15$ would need to be increased by factors of 3 to 5 . Until more definitive data concerning this $M$ star population are available, until Luyten's massive campaign to provide reliable data for some 500000 stars with annual proper motions larger than $0 \% 1$ is finished, and until large numbers of trigonometric parallaxes are provided by the program at the U.S. Naval Observatory, the present stellar luminosity function had best be left unchanged.

At La Plata Observatorio Astronómico a rediscussion of the stellar luminosity function based upon trigonometric parallax methods has been finished by Muzzio. The luminosity function derived is systematically lower than that of Luyten (1968), and shows a rather close agreement with the luminosity function of the Hyades cluster.

Luminosity functions for the stellar population within $25 \mathrm{pc}$ of the Sun have been published by Woolley et al. (06.155.007). For all stars within this volume $\log \phi\left(M_{v}\right)$ is in good agreement with the standard van Rhijn function for $+4<M_{\mathrm{v}}<+10$. The authors also give luminosity functions 
for this range in $M_{\mathrm{v}}$ for "all stars, corrected for box angle; young stars; and young stars, corrected for box angle." A review paper by van de Kamp (06.155.008) giving the characteristics of the stellar population within $5 \mathrm{pc}$ of the Sun includes a luminosity function which has a broad maximum from $M_{\mathrm{v}}=+10$ to +15 .

Cudworth (07.153.016) has investigated the field star population near NGC 2168 (M35) by means of $U B V$ photometry and proper motion data. The luminosity function for a field in Vela at $l=281^{\circ}$, $b=+399$ has recently been investigated by Wooden (1971). Similar studies in LF $12\left(l=241^{\circ}\right.$, $b=-0.5)$ and LF $14\left(l=298^{\circ}, b=+1.4\right)$ are in progress at the Warner and Swasey Observatory.

$R G U$ photometry has been applied by Fang (1968) to construct the stellar luminosity function $\log \phi\left(M_{\mathrm{G}}\right)$ for $+1<M_{\mathrm{G}}<+7$ in a region at $l=162^{\circ}, b=-0.4$. From $R G U$ photometry of a field at the border of the Scutum cloud $(l=25 \% 4, b=-4.4)$ Schubart (04.113.025) has determined a luminosity function for absolute $G$ magnitudes in the range $+3<M_{G}<+6$. A similar study by Fünfschilling (06.113.003) of a field at $l=1: 2, b=-3.6$ in the Large Sagittarius Cloud also yielded luminosity functions at distances of 0.4 to $0.65 \mathrm{kpc}$ from the Sun.

Hughes (1972) has devised an extension of the method originally used by Oort for determining either the space density or the luminosity function from star counts covering large areas of the sky. The space density is assumed to vary only in the direction perpendicular to the galactic plane. He has applied the method to some 1071 stars with I-K color indices between 1.50 and 2.50 as published in the Two-Micron Sky Survey: A Preliminary Catalogue (Neugebauer and Leighton, 1969). This range in color index includes spectral types G5 to M2 approximately, with the predominant spectral classes at $\mathrm{K} 0$ to $\mathrm{K} 5$. The method is promising for ultimate compilation of a general luminosity function appropriate to star counts at $2 \cdot 2 \mu$ (K magnitudes).

\section{REFERENCES}

Bell, R. A., FitzGerald, M. P. $\quad$ 1971, in M. Hack (ed.) Coll. on Supergiant Stars, Trieste, p. 168. Bouw, G. D., Parsons, S. B. 1971, in M. Hack (ed.), Coll. on Supergiant Stars, Trieste, p. 22. Buscombe, W. $\quad 1972, M t$. Stromlo Mimeograms No. 8, Mt. Stromlo Obs. Canberra, ACT.

Code, A. D. 1972, The Scientific Results from the Orbiting Astronomical Observatory (OAO 2); NASA SP-310, National Aeronautics and Space Administration, Washington, D.C.

Crawford, D. L. $\quad 1972$, IAU Symp. No. 54 (in preparation).

Crézé, M. 1972, Astron. Astrophys., 21, 85.

Fang, C. C.-Y. 1968, Ph. D. Thesis, Univ. Pennsylvania, Philadelphia, Pa.

Habing, H. J., Heiles, C. E. $\quad$ 1971, IAU Trans., 14B, 220.

Hughes, E. E. 1972 , Astron. J. (in press).

Johnson, H. L. $\quad$ 1966, Ann. Rev. Astron. Astrophys., 4, 193.

Johnson, H. L. $\quad 1968$, Stars and Stellar Systems, 7, 167.

Luyten, W. J. 1968, Monthly Notices Roy. Astron. Soc., 139, 221.

Lyngå, G., Hansson, N. 1972, Astron. Astrophys. Suppl. Ser., 6, 327.

Neugebauer, G., Leighton, R. B. $\quad$ 1969, Two-Micron Sky Survey: A Preliminary Catalogue; NASA SP3047, National Aeronautics and Space Administration, Washington, D.C.

Smith, L. F. $\quad$ 1968, Monthly Notices Roy. Astron. Soc., 140, 409.

Stothers, R. $\quad$ 1972, Publ. Astron. Soc. Pacific., 84, 373.

Tuve, M. A., Lundsager, S. $\quad 1972 a$, Astron. J., 77, 652.

Tuve, M. A., Lundsager, S. 1972b, Carnegie Institution Monograph, Carnegie Institution of Washington (in press).

Upgren, A. R., Grossenbacher, R., Penhallow, W. S., MacConnell, D. J., Frye, R. L. $\quad$ 1972, Astron. J., $77,486$.

Wawrukiewicz, A. S. $\quad 1970$, Ph. D. Thesis, Univ. Rochester, Rochester, N.Y.

Weaver, H. F., Ebert, A. $\quad$ 1964, Publ. Astron. Soc. Pacific, 76, 6.

Weaver, H. F. 1972, in B. J. Bok, C. S. Cordwell, R. M. Humphreys (eds.), Problems of Galactic Spiral Structure, Steward Obs., Tucson, Arizona, p. 28.

Westerhout, G. 1973, Maryland-Green Bank Galactic 21-cm Line Survey, 3rd Edition, Univ. Maryland, College Park, Md.

Wooden, W. H. II. 1971, Publ. Warner and Swasey Obs., 1, No. 2. 